\section{Biology is the new physics}

\author{
The increasing use of mathematics in biology is both inspiring \\ research in mathematics and creating new career options for \\ mathematical biologists
}

\section{Philip Hunter}

B iology has long been the stepchild of the natural sciences. Compared with mathematical proofs, physical formulae and the molecules of chemistry, biology, like life itself, has often seemed unquantifiable, unpredictable and messy. Yet, scientists have striven gallantly to pin biology down through the application of the other natural sciences. Ever since Gregor Mendel (1822-1884) formulated the laws of heredity, biology has slowly transformed itself into a 'hard' science. In fact, research in biology and medicine depends increasingly on mathematics and computation, with profound consequences for those fields and for the skills needed to conduct research and clinical development. The conversion of biology into a more quantifiable science will continue to the extent that it might even become the main driving force behind innovation and development in mathematics. This, at least, is the view of Michael Mackey, President of the Society for Mathematical Biology, which aims to foster international interactions between the two fields.

\section{The conversion of biology into a more quantifiable science will continue to the extent that it might even become the main driving force behind innovation and development in mathematics}

\footnotetext{
"For many years the inspiration for innovation in applied mathematics has come from physics, but in my opinion, in this century it will come from the biological sciences, broadly defined," Mackey explained, adding that this switch has been taking place slowly over several decades. While physics has stagnated, waiting for new theoretical insights to make progress against fundamental problems such as quantum gravity, Mackey argued, theoretical biology has emerged as a new source
}

of inspiration for mathematicians. "In my 40 plus years of research, I have found that problems in biomathematics almost always uncover unexplored and undeveloped areas of mathematics," he said. "These are areas that mathematicians have not even thought about exploring. New mathematics."

n some cases, new mathematical techniques have been developed to tackle specific problems, but biology has also stimulated fundamental progress in mathematics, according to Jürgen Jost, Director of the Max Planck Institute for Mathematics in the Sciences in Leipzig, Germany. Jost cited the field of information geometry, which first emerged as a method to combine geometry and statistical probability theory to tackle problems in other scientific fields including physics and economics. "The new field of information geometry draws a lot [of] inspiration from information processing in biological systems, from the cell to the brain," he explained.

Information geometry is increasingly applied across a range of biology disciplines at different scales of time and space. For example, in proteomics, it is used to analyse interactions between macromolecular complexes; in modelling epidemics, it is able to account for more complex and subtle differences in quantities, such as rates of infection among populations, than previous approaches. Indeed, the reason for the growing use of information geometry across biological and medical research is that it is capable of allowing for non-uniformity in the systems under study, whether at the scale of a protein, cell, pathway or ecosystem. In essence, information geometry combines geometry with probability theory to model changing, complex and nonlinear systems.

Other techniques have evolved alongside information geometry to analyse interactions between processes that operate across a huge range of spatiotemporal scales. The human immune system, for example, involves events at the level of individual gene expression or protein interactions that take place in nanoseconds; cellular mechanisms or body-wide events that can take minutes, hours or days; and interactions between people or even whole populations that last months and years. Trying to understand these systems involves bridging different layers and scales, and this need, Jost points out, has driven developments in mathematical biology.

T he application of mathematics to biology itself is hardly new and dates back at least to Mendel and his work on the inheritance of traits in the midnineteenth century. This led eventually to the theory of Mendelian inheritance as the foundation of modern genetics for all sexually reproducing organisms, which assumes that traits are passed down as whole units on a digital basis, rather than being watered down in an analogue fashion. In this case, the mathematics was straightforward: simple probability theory applied to trait inheritance. The difference in the present relationship between biology and mathematics is that there is now a two-way trade in ideas and research, which leads to more complex tools and applications in and across both sciences.

\section{...mathematical biology is now spreading beyond fundamental questions and is starting to yield practical benefits}

Another difference is that mathematical biology is now spreading beyond fundamental questions and is starting to yield practical benefits. It is being applied widely in cancer research, for instance, to both model the growth and metastasis of tumours and to understand why treatment sometimes fails. According to Mark Chaplain, Head of the Mathematics Division at the University of Dundee in the UK, the application of mathematical techniques has led to substantial progress in three key areas of cancer research.

The first is a series of developments in modelling tumour-induced angiogenesisthe process by which new blood vessels are formed as the tumour grows. This was previously poorly understood and, according to Chaplain, involves irregular smallscale processes that cannot be tackled with 




simple linear models. "This began with a paper in 1998 featuring a mathematical modelling technique to capture the growth of new blood-vessels," he said (Anderson \& Chaplain, 1998). Since then, the field has advanced in various ways to model changes in the dynamics of the blood system as the tumour grows, and to include delivery of drugs in the models (McDougall et al, 2010).

The second area concerns the process of metastasis or the invasion by malignant cells of tissues elsewhere in the body. "For similar reasons to angiogenesis-that is, the complexity of cancer and the number of nonlinear feedback mechanisms-it is very difficult to gain precise quantitative and predictive information about how many types of cancers grow and spread," Chaplain explained. "Using mathematical modelling, we can begin to quantify not only growth but also metastatic spread." Most recent models include the vital process known as transendothelial migration, by which a malignant cell detaches itself from the tumour and breaks through into the bloodstream so that it can be transported to a distant part of the body. This requires the cell to have suitable biophysical properties, but also calls for assistance from adhesion molecules that enable the cell to attach itself temporarily to the inner wall of a blood or lymph vessel and create a gap through which it then enters the bloodstream or lymphatic system (Ramis-Conde et al, 2009).

The third big application of mathematical biology in cancer research is more directly related to therapies: it is used to model radiotherapy regimes and understand why cancers sometimes return. "We have suggested a mechanism explaining why breast cancer recurs in a certain percentage of women even after surgical treatment and standard radiotherapy treatment," Chaplain said. "This was only possible through the computational simulation results of the model" (Enderling et al, 2007). Chaplain believes that research in all three of these fields will lead to therapeutic advances within the next 5-10 years.



aturally, this growing role of mathematics in many research projects has significant implications for the skills required and the teams involved. An obvious question is whether there is a need for people trained in a discipline called mathematical biology, or whether the call ought to be for better cooperation between mathematicians and biologists. Chaplain is in little doubt that the overwhelming need is to attract good mathematicians in the first instance - those who might have started out not considering biology at all. "Personally, I am looking for talented applied mathematics students with an interest in working in an inter-disciplinary environment," he said. "In my opinion, it will always be the case that it is easier for mathematicians to learn the biology rather than the other way round."

\section{...this growing role of mathematics in many research projects has significant implications for the skills required and the teams involved}

A similar line is taken by Xavier Salvatella, Principal Investigator at the Laboratory of Molecular Biophysics at the Institute for Research in Biomedicine in Barcelona, Spain, which has completed pioneering work on the computational modelling of protein movement. He argues that the strongest mathematical biology teams are composed of people whose skills complement one another, rather than of 'all-rounders' who may be quite good at everything but excel at nothing. "In my opinion, teams that are truly multidisciplinary are more productive and creative than teams formed by 
multidisciplinary individuals," Salvatella explained. "A solid training in a core science (physics, computer science, chemistry, biology) or maths is a key asset, as is an interest in different fields."

\section{...there is also a growing need for truly multidisciplinary individuals for recruitment to small projects that are not able to resource separate people with each of the skills needed}

Jost also pointed out that there is a need for all members of a team to be interested in and to acquire some understanding of other disciplines in order to communicate and collaborate effectively. "Biologists can learn mathematics and some of the very bright students of the new generation are presently doing that, but this will not work with the majority of biologists," he said. "Conversely, mathematicians can learn biology-that is what I try to teach my students-and in my group, this works well." Jost added that there will always be a need for people with more traditional experimental skills to collect the data.

$\mathrm{H}$ owever, there is also a growing need for truly multidisciplinary individuals for recruitment to small projects that are not able to resource separate people with each of the skills needed, according to Stephen Eglen, who specializes in computational modelling of the development of the nervous system at the University of Cambridge, UK. "Clearly there is a need for more collaborations between biologists and mathematicians, especially for large-scale efforts," he said. "But for smaller projects, I think it is incredibly useful to train one person to be competent in both the experimental and theoretical aspects of a problem. Hence I do think it important for biologists to get more theoretical training, and vice-versa for mathematicians."

Doing so, however, is easier said than done. "The major difficulty in computational neuroscience seems to be to train people to find the middle ground, that is theoreticians with a true interest and respect for the biology, or biologists with a true theoretical interest," commented Michael Brecht from the Bernstein Center for Computational Neuroscience in Berlin, Germany. "If either of [these] is achieved, the initial background is less important."

To this end, the University of Cambridge has created an 11-month Master of Philosophy degree in bioinformatics, which combines computational studies with biology. The programme is aimed at students who trained in any of biology, mathematics, computer science, or possibly physics and who want to learn sufficient biology for a subsequent PhD course in bioinformatics, or a career in industry. "The initial aim was to take both biology and maths graduates," Eglen explained. "Over the last six years of the course, we've mostly taken maths graduates, and teaching both biologists and theorists in the same class is a challenge." At present, only $10-20 \%$ of the course students come from a biological background, which suggests that mathematical biology is having some success in winning hearts and minds, at least among non-biologists. However, it less clear whether it is sufficient to convert pure biology graduates into successful mathematical biologists, unless they have at least done some maths during their degree courses.

M eanwhile, the growing number of significant advances made in the realm of theoretical biology could enhance the appeal of the field to new undergraduates and graduates. Perhaps the most exciting of these developments was the recent discovery of the vital role played by quantum mechanics in photosynthesis, arguably the most important set of chemical reactions on the planet. Photosynthesis exploits a phenomenon known as quantum coherence that involves synchronization between distant molecules. In photosynthesis, quantum coherence synchronizes the pigment molecules that harvest sunlight in a way such that the light energy is transmitted more quickly from the surface to the inner reaction centres, thereby reducing the time available for energy to be lost as heat or radiated back out as light during the transfer process (Collini et al, 2010). In this way, quantum coherence boosts the efficiency of light harvesting in photosynthesis.

\section{...mathematical biology itself can appeal to people who are interested in either a theoretical or practical career in almost any discipline...}

The broader point is that mathematical biology itself can appeal to people who are interested in either a theoretical or practical career in almost any discipline, whether this is cancer research, disease control, environmental issues, or fundamental questions concerning genetics and evolution. In any case, mathematics is quickly becoming as integral to biology as it is to physics, and ought therefore to deserve more attention in the education and training of biologists at all levels.

\section{REFERENCES}

Anderson ARA, Chaplain MAJ (1998) Continuous and discrete mathematical models of tumourinduced angiogenesis. Bull Math Biol 60: 857-899

Collini E, Wong CY, Wilk KE, Curmi PMG, Brumer P, Scholes GD (2010) Coherently wired light-harvesting in photosynthetic marine algae at ambient temperature. Nature 463: 644

Enderling $\mathrm{H}$, Chaplain MAJ, Anderson ARA, Vaidya J (2007) A mathematical model of breast cancer development, local treatment and recurrence. / Theor Biol 246: 245-259

McDougall SR, Chaplain MAJ, Stéphanou A, Anderson ARA (2010) Modelling the impact of pericyte migration and coverage of vessels on the efficacy of vascular disrupting agents. Math Model Nat Phenom 5: 163-202

Ramis-Conde I, Chaplain MAJ, Anderson ARA Drasdo D (2009) Multi-scale modelling of cancer cell intravasation: the role of cadherins in metastasis. Phys Biol 6: 016008

Philip Hunter is a freelance journalist in London, UK.

EMBO reports (2010) 11, 350-352.

doi:10.1038/embor.2010.55 